\title{
Assessment of global DNA methylation in the first trimester fetal tissues exposed to maternal cigarette smoking
}

\author{
Svetlana $\mathrm{Fa}^{1,2 \dagger}{ }^{1}$, Trine Vilsbøll Larsen ${ }^{1 \dagger}$, Katrine Bilde ${ }^{1}$, Tina F. Daugaard ${ }^{1}$, Emil H. Ernst ${ }^{1}$, Rasmus H. Olesen ${ }^{1}$,
} Linn S. Mamsen ${ }^{3}$, Erik Ernst ${ }^{1,4}$, Agnete Larsen $^{1 \dagger}$ and Anders L. Nielsen ${ }^{1{ }^{*+}}$ (D)

\begin{abstract}
Aims: Maternal cigarette smoking during pregnancy increases the risk of negative health consequences for the exposed child. Epigenetic mechanisms constitute a likely link between the prenatal exposure to maternal cigarette smoking and the increased risk in later life for diverse pathologies. Maternal smoking induces gene-specific DNA methylation alterations as well as global DNA hypermethylation in the term placentas and hypomethylation in the cord blood. Early pregnancy represents a developmental time where the fetal epigenome is remodeled and accordingly can be expected to be highly prone to exposures with an epigenetic impact. We have assessed the influence of maternal cigarette smoking during the first trimester for fetal global DNA methylation.

Methods and results: We analyzed the human fetal intestines and livers as well as the placentas from the first trimester pregnancies. Global DNA methylation levels were quantified with ELISA using a methylcytosine antibody as well as with the bisulfite pyrosequencing of surrogate markers for global methylation status, LINE-1, and AluYb8. We identified gender-specific differences in global DNA methylation levels, but no significant DNA methylation changes in exposure responses to the first trimester maternal cigarette smoking.

Conclusions: Acknowledging that only examining subsets of global DNA methylation markers and fetal sample availability represents possible limitations for the analyses, our presented results indicate that the first trimester maternal cigarette smoking is not manifested in immediate aberrations of fetal global DNA methylation.
\end{abstract}

Keywords: Epigenetics, DNA methylation, Smoking, Xenochemicals, Toxicology

\section{Introduction}

Prenatal exposure to maternal cigarette smoking (PEMCS) represents a fetal exposure with consequences for the birth weight and delivery term $[1,2]$. PEMCS also predisposes individuals for diseases later in life [3-5]. This includes reduced pulmonary function and increased asthmatic symptoms in childhood [6-9], changes in children's neurodevelopment and behavior [10-12], an increased incidence of childhood obesity and metabolic disorders [13-15], and reduced cardiovascular health among children [16-19]. In the Western world, approximately $25 \%$ of women of fertile age are smoking cigarettes and $7 \%$

\footnotetext{
* Correspondence: aln@biomed.au.dk

${ }^{\dagger}$ Equal contributors

'Department of Biomedicine, Aarhus University, Bartholin building, DK-8000

Aarhus C, Denmark

Full list of author information is available at the end of the article
}

continue smoking throughout pregnancy [4]. Epigenetics, at least in part, may explain the connection between PEMCS and increased disease risk later in life $[3,20]$. Epigenetics can mechanistically describe the regulation of cellular gene expression in response to a given environment, with epigenetics functioning through short and long non-coding RNA (ncRNA), chromatin remodeling, histone modification, and DNA methylation. DNA methylation patterns can be dynamic, display the cell type and tissue specificity, and change upon environmental exposure [21-23]. The DNA methylation profile in the zygote is reprogrammed during the cleavage phase with massive de novo DNA methylation following the implantation phase [24, 25]. Stringent-controlled dynamics for removing DNA methylation and de novo DNA methylation is essential for correct development, with early 
embryogenesis representing one critical window in which environmental factors can influence DNA methylation in offspring $[22,23]$. PEMCS is shown to induce quantitative alterations in position-specific $\mathrm{CpG}$ methylation in the placenta and blood from newborns, and DNA methylation changes can be maintained into adulthood [3, 20, 26-34]. The latter are exemplified in the longitudinal studies by Richmond et al. and Lee et al., in which the researchers collected peripheral blood samples and examined the methylation status of $\mathrm{CpG}$ sites manifesting PEMCS-induced DNA methylation changes in the cord blood at birth [30, 34]. The longitudinal analyses at age 17 years showed persistently changed patterns of DNA methylation for CpG sites in AHRR (cg05575921), MYO1G (cg22132788), CYP1A1 (cg09935388), and CNTNAP2 (cg25949550), whereas the reversibility of DNA methylation was observed for CpG sites in GFI1 (cg09935388), KLF13 (cg26146569), and ATP9A (cg07339236) [30, 34].

Similar to locus-specific DNA methylation changes, global DNA methylation changes also represent a biodosimeter of lifelong environmental exposures [35]. The consequence of PEMCS for global methylation was addressed by Wilhelm-Benartzi et al., who in placenta tissue examined both gene-associated loci and long interspersed nuclear element-1 (LINE-1) and AluYb8 repetitive elements [36]. LINE and Alu repeat elements act as surrogate markers for global DNA methylation measurements $[36,37]$. In the term placentas, the DNA methylation level of $A l u Y b 8$ was significantly higher among infants prenatally exposed to cigarette smoke, whereas no significant DNA methylation changes were observed for LINE-1 and gene-associated loci [36]. Another study of term placenta samples also failed to find any significant association between LINE-1 DNA methylation and PEMCS [38] but, notably, gene-associated CpG site-specific DNA methylation alterations have been described in the placenta $[28,33,39,40]$. In the cord blood, using methyl-specific ELISA-based methodology, global DNA hypomethylation was observed in newborns from PEMCS and second-hand smoking exposure [41]. For LINE-1, no significant change in DNA methylation in the cord blood was observed between cigarette smoke-exposed and non-exposed children, but an association between LINE-1 DNA methylation status and birth weight was present [38]. In buccal cells from children with PEMCS, AluYb8 hypomethylation was observed, whereas in LINE-1 DNA methylation was not significantly affected [42].

Even if PEMCS is described to induce global alterations in DNA methylation present at the time of birth and such changes, at least to some extent, can be maintained into later life, to our knowledge no descriptions are present of the timing for the developmental onset of
PEMCS-mediated global DNA methylation changes. Accordingly, we examined whether PEMCS-induced global DNA methylation changes are manifested already during early fetal development, a period expected to be particularly prone to exposure-induced epigenetic alterations.

\section{Materials and methods \\ Ethics statement and sample collection}

The fetal tissues were obtained with informed consent from women seeking a legal (<gestation week 12) abortion in a regional hospital within Region Midtjylland, Denmark. The study was approved by The Danish National Committee on Health Research Ethics (approval no. KF (01)258206), and all the experiments were performed in accordance with the Helsinki Declaration. There was no change in treatment or care associated with recruitment to the study. All personal identification data are anonymized. Information concerning smoking habits was obtained. Cotinine concentrations were previously measured in maternal serum and fetal organs for a similar sample cohort, and the observed correspondence between reported smoking status and cotinine concentration supported the reliability of the women's selfreported smoking habits [43]. Fetal tissue (6 to 12 weeks of pregnancy) was surgically removed from the uterus according to routine procedures. The age of the fetuses was determined by ultrasound examination prior to the procedure. Immediately after the surgical procedure, placental and fetal tissues were rinsed in sterile isotonic saline and carefully dissected under a stereomicroscope. All fetuses appeared morphologically normal, and no disease was suspected prior to the procedure. The small intestine, liver, and placenta samples were isolated, rinsed in sterile isotonic saline and placed in separate tubes containing RNA-later (Ambion, Inc., Austin, TX, USA). The samples were stored for $2-4$ hours at room temperature and then frozen at $-20{ }^{\circ} \mathrm{C}$.

Tissue biopsies were snap frozen in RNA-later (Ambion, Inc., Austin, TX, USA). The sample collection was continuously expanded throughout the study period, and samples representing each tissue and experimental assay are not systematically the same. The numbers of samples for each tissue $(N)$, subdivided into female (f) and male (m) samples, or smoking (S) and non-smoking (NS) samples, were $N=40$, with $19 \mathrm{f}$ and $21 \mathrm{~m}$ and $17 \mathrm{~S}$ and $23 \mathrm{NS}$, for the placenta; $N=33$, with $14 \mathrm{f}$ and $19 \mathrm{~m}$ and $18 \mathrm{~S}$ and $15 \mathrm{NS}$, for the liver; and $N=21$, with $15 \mathrm{f}$ and $6 \mathrm{~m}$ and 12 $\mathrm{S}$ and $9 \mathrm{NS}$, for the small intestine. More details on the samples used for each particular experimental assay including information on fetal gender, age, and smoking status of the mothers are described in Additional file 1: Table S1 as well as in the figure legends for the given experimental settings. Differences in the age distribution for males and females, as well as for smoking-exposed or 
non-smoking-exposed groups, were, if present, encountered in subsequent data analyses.

\section{DNA extraction and gender determination}

DNA was extracted using the MasterPure ${ }^{\text {tm }}$ Complete DNA and RNA Purification Kit (Epicentre, Madison, WI, USA). DNA quantities were measured using a Nanodrop spectrophotometer (Thermo Scientific, Wilmington, USA). Gender was determined by pyrosequencing, as previously described [44].

\section{ELISA-based global DNA methylation quantification}

The percentage of methylation in total DNA was determined by measuring 5 -methylcytosine $(5-\mathrm{mC})$ using a 5 mC DNA ELISA kit (Zymo Research Corp, Orange, CA, USA) that features a unique anti-5-mC monoclonal antibody that is both sensitive and specific for 5-mC. Assays were performed according to the manufacturer's instructions with the modified standard curve. The percentages of prepared methylated DNA standards were 5, 1.66, 0.55 , and $0.185 \%$. The amount of total DNA used was $100 \mathrm{ng}$. Logarithmic second-order regression was used to calculate the results. Since the positive and negative controls used to prepare the standard curve consist of Escherichia coli gDNA, the obtained results were multiplied by 5.68 to correct for fold differences in the density of CpGs between E. coli and humans following the recommendations from the manufacturer.

\section{Bisulfite conversion and pyrosequencing for global DNA methylation analyses}

A total of $1.2 \mu \mathrm{g}$ of the small intestine and $2 \mu \mathrm{g}$ of the placenta and liver genomic DNA was bisulfite-converted using the EpiTect Bisulfite Kit (Qiagen, Hilden, Germany) according to the manufacturer's instructions. Specific genomic DNA sites were amplified by PCR using $5 \mu \mathrm{l}$ of bisulfite-converted genomic DNA as templates. PCR reactions were performed using PyroMark PCR kit (Qiagen) according to the manufacturer's instructions. Pyrosequencing for quantitative DNA methylation analyses was performed using the PyroMark Q24 Advanced system (Qiagen). LINE-1 amplification and pyrosequencing was performed using a predesigned assay PyroMark Q24 CpG LINE-1 (Qiagen). AluYb8 amplification and pyrosequencing was performed as previously described [37].

\section{Statistics}

An unpaired $t$ test was used to compare two sample groups, and a comparison between multiple groups was done using two-way (gender and smoking exposure) analysis of variance (two-way ANOVA) using the GraphPad Prism (GraphPad Software, La Jolla, CA, USA). Analysis of covariance (ANCOVA) was used to examine the interaction between smoking, gender, and age, where age was treated as a continuous exploratory variable and smoking status and gender were treated as factors. ANCOVA was carried out using $\mathrm{R}$ version 3.3.1, and the outcomes are presented in Table 1 . The normal distribution of data was investigated by QQ-plot, whereas the linearity and the homoscedasticity of residuals were assessed by inspecting a plot of the residuals against the explanatory variables. We did not observe any violation of the assumptions for the analyses. In all analyses, values of $p<0.05$ were considered statistically significant.

\section{Results}

Placenta global DNA methylation levels and PEMCS

To characterize the impact of PEMCS for global DNA methylation on fetuses and placentas, we sampled the

Table 1 ANCOVA for the effects of fetal age, gender, and PEMCS for DNA methylation levels in the first trimester placentas, fetal livers, and small intestines

\begin{tabular}{|c|c|c|c|c|}
\hline Tissue & Assay & Variable & $F$ value & $P$ value \\
\hline \multirow[t]{9}{*}{ Placenta } & \multirow[t]{3}{*}{ 5-mC ELISA } & Age & 8.567 & $0.008^{*}$ \\
\hline & & Gender & 8.434 & $0.008^{*}$ \\
\hline & & PEMCS & 0.704 & 0.411 \\
\hline & \multirow[t]{3}{*}{ AluYb8 } & Age & 0.059 & 0.811 \\
\hline & & Gender & 4.527 & $0.042^{*}$ \\
\hline & & PEMCS & 2.958 & 0.096 \\
\hline & \multirow[t]{3}{*}{ LINE-1 } & Age & 0.376 & 0.544 \\
\hline & & Gender & 0.013 & 0.909 \\
\hline & & PEMCS & 1.017 & 0.320 \\
\hline \multirow[t]{9}{*}{ Liver } & \multirow[t]{3}{*}{ 5-mC ELISA } & Age & 1.214 & 0.286 \\
\hline & & Gender & 0.191 & 0.667 \\
\hline & & PEMCS & 0.001 & 0.977 \\
\hline & \multirow[t]{3}{*}{ AluYb8 } & Age & 3.190 & 0.085 \\
\hline & & Gender & 0.004 & 0.953 \\
\hline & & PEMCS & 0.106 & 0.747 \\
\hline & \multirow[t]{3}{*}{ LINE-1 } & Age & 0.263 & 0.612 \\
\hline & & Gender & 8.779 & $0.006^{*}$ \\
\hline & & PEMCS & 0.672 & 0.419 \\
\hline \multirow[t]{9}{*}{ Small intestine } & \multirow[t]{3}{*}{ 5-mC ELISA } & Age & 3.576 & 0.078 \\
\hline & & Gender & 0.445 & 0.515 \\
\hline & & PEMCS & 0.147 & 0.707 \\
\hline & \multirow[t]{3}{*}{ AluYb8 } & Age & 0.006 & 0.942 \\
\hline & & Gender & 1.417 & 0.250 \\
\hline & & PEMCS & 0.121 & 0.733 \\
\hline & \multirow[t]{3}{*}{ LINE-1 } & Age & 0.327 & 0.575 \\
\hline & & Gender & 0.568 & 0.462 \\
\hline & & PEMCS & 1.783 & 0.201 \\
\hline
\end{tabular}

Analyses were done with age as a continuous variable and gender and PEMCS as factors

*Indicates statistical significance, $p<0.05$ 
human placentas, fetal livers, and fetal small intestines representing days 44 to 82 post-gestation. Gender was determined by pyrosequencing a polymorphic region of the amelogenin gene, as previously described [45]. Smoking status was assigned to mothers with self-reported daily smoking (approximately 90\% of the smoking mothers reported smoking 6-20 cigarettes per day). In addition, information regarding exposure to passive smoking was obtained. Whereas cigarette smoking mothers also reported exposure to passive smoking, this additional exposure was not reported to be present among the non-smoking mothers. A description of the gender, age, and smoking status of the study samples is presented in Additional file 1: Table S1.

To address whether PEMCS manifests global DNA methylation alterations during the first trimester in the placenta, we first performed an ELISA methylcytosine antibody-based measurement of global DNA methylation (5-mC ELISA). No statistically significant effects of PEMCS were observed for the global DNA methylation level in the placenta (Fig. 1a). Gender-specific DNA methylation quantitative effects of PEMCS have been described [46-48], and accordingly, a two-way ANOVA was performed to investigate the impact of PEMCS and gender on DNA methylation levels. We observed that lower methylation levels were present in the placenta from female pregnancies than male pregnancies $(p=0.004$, two-way ANOVA) (Additional file 2: Figure S1A). To investigate whether fetal age affects DNA methylation levels in the placenta, we performed ANCOVA with age, gender, and PEMCS status as variables (Table 1). This analysis identified a statistically significant increase in global DNA methylation with age $(p=0.008)$ (Table 1). Altogether, the ELISA analyses showed that global DNA methylation levels in the placenta are affected by fetal age and gender but not by PEMCS.
The average DNA methylation level for repetitive $A l u Y b 8$ elements represents an estimate for the global DNA methylation level [37]. By measuring AluYb8 DNA methylation levels in term placenta, Wilhelm-Benartzi et al. identified that PEMCS resulted in hypermethylation (66.2\% in PEMCS exposed versus 64.8\% in non-exposed placenta tissue) [36]. We analyzed our samples with an AluYb8 bisulfite pyrosequencing assay similar to the assay used by Wilhelm-Benartzi et al. [36]. The AluYb8 bisulfite pyrosequencing assay measures the DNA methylation status of five consecutive $\mathrm{CpG}$ sites. For each tested DNA sample, we determined the average DNA methylation level for these five CpG sites. A comparison between smoking-exposed and non-exposed samples revealed that PEMCS did not induce alterations in AluYb8 DNA methylation (Fig. 1b). We next questioned whether differences in DNA methylation levels existed between smoking-exposed and non-exposed samples at individual AluYb8 CpG sites. We detected no PEMCS-induced changes in DNA methylation levels for any of the five CpG sites (Additional file 2: Figure S1B). Two-way ANOVA using the DNA methylation data for the average $A l u Y b 8$ DNA methylation level for each sample identified a lower AluYb8 DNA methylation level in females than males ( $p=0.036$, two-way ANOVA) (Additional file 2: Figure S1C). We note that AluYb8 methylation levels in term placentas previously were described to be gender dependent [36]. A subsequent ANCOVA revealed no impact of the fetal age on AluYb8 DNA methylation in placentas (Table 1). Similar to $A l u Y b 8$, the average DNA methylation level for repetitive LINE-1 elements represents an estimate for the global DNA methylation level [37]. We analyzed our samples with a LINE-1 bisulfite pyrosequencing assay. The used LINE-1 bisulfite pyrosequencing assay measures DNA methylation levels for three consecutive CpG
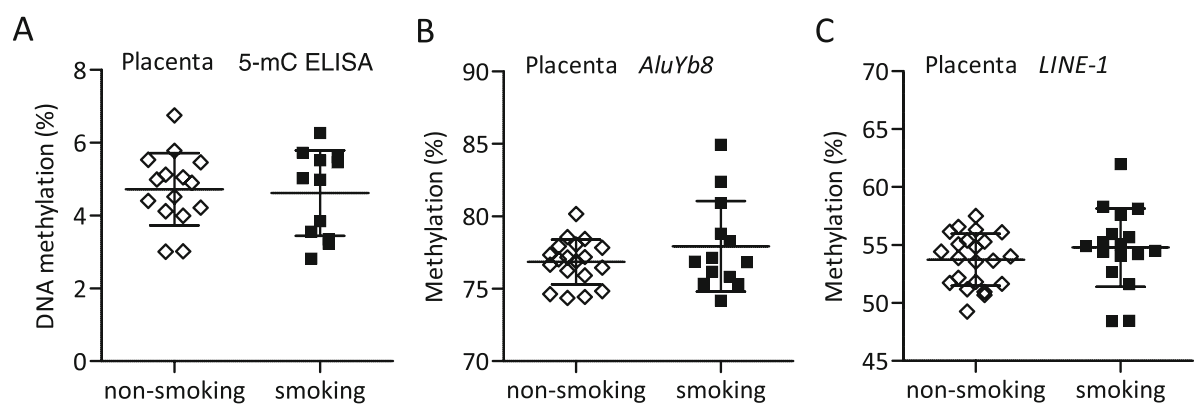

Fig. 1 Global DNA methylation in placentas. DNA methylation determined by ELISA using a 5-mC-specific antibody (non-smoking group $N=15$ and smoking group $N=12$ ). Data represent the means of two experiments. The average DNA methylation percentage for $\mathbf{b}$ five consecutive AluYb8 CpG sites (non-smoking group $N=19$ and smoking group $N=13$ ) and $\mathbf{c}$ three consecutive LINE-1 CpG sites (non-smoking group $N=22$ and smoking group $N=17$ ) were determined by pyrosequencing bisulfite-converted DNA. Data represent the means of two runs, except for samples that differed more than 5\% in methylation levels within the duplicates. For such samples, a third run was performed and the presented data represent the means of the three runs. Mean methylation percentages \pm SD are shown. Unpaired $t$ test was used to compare two groups. A $p$ value $<0.05$ was considered statistically significant 
sites. For each tested DNA sample, we determined the average methylation level of all three CpG sites. A comparison between smoking-exposed and non-exposed samples revealed that PEMCS did not induce alterations in LINE-1 DNA methylation (Fig. 1c). We next questioned whether differences in DNA methylation levels existed between smoking-exposed and non-exposed samples at individual LINE-1 CpG sites. We detected no PEMCS-induced changes in DNA methylation levels for any of the three $\mathrm{CpG}$ sites (Additional file 2: Figure S1D). Moreover, two-way ANOVA did not detect gender differences in LINE-1 DNA methylation levels (Additional file 2: Figure S1E), and ANCOVA revealed no association between LINE-1 DNA methylation levels and fetal age (Table 1). From the described analyses of surrogate repetitive element markers, we conclude that PEMCS in the first trimester is not manifested in placenta global DNA methylation changes and no interaction is present between PEMCS, gender, and age (data not shown).

\section{Global methylation levels in fetal livers and small intestines and PEMCS}

We next questioned whether global changes in DNA methylation were present in the fetus due to the first trimester PEMCS. We first examined global quantitative DNA methylation levels in the fetal livers, which represent a key metabolic target tissue. In a 5-mC ELISA- based DNA methylation analysis, we identified no significant differences caused by PEMCS (Fig. 2a). Twoway ANOVA revealed a similar level of DNA methylation in males and females (Additional file 3: Figure S2A) and no impact of fetal age for the global DNA methylation level in the fetal livers were detected by ANCOVA (Table 1). We next addressed AluYb8 DNA methylation with the same bisulfite pyrosequencing assay used for the placenta analysis. PEMCS was not identified to have an impact on the average DNA methylation level of the five AluYb8 CpG sites tested (Fig. 2b). DNA methylation levels for the individual AluYb8 CpG sites also were unaffected by PEMCS (Additional file 3: Figure S2B). Gender was not identified to have an impact on $A l u Y b 8$ DNA methylation (Additional file 3: Figure S2C). AluYb8 DNA methylation levels were not affected by the age of the fetal liver samples (Table 1, ANCOVA). LINE-1 DNA methylation levels were next measured with the same bisulfite pyrosequencing assay used for placenta samples. No significant effects of PEMCS were observed for LINE-1 methylation, either for the average DNA methylation level for the three LINE-1 CpG sites tested or for each of the three individual $\mathrm{CpG}$ sites (Fig. 2c, Additional file 3: Figure S2D). We detected significantly lower LINE-1 DNA methylation levels in females compared to that in males $(p=0.009$, two-way ANOVA) (Additional file 3: Figure S2E). LINE-1 DNA

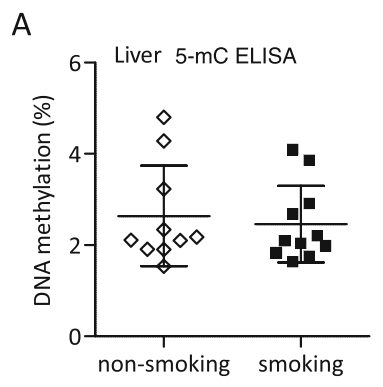

D

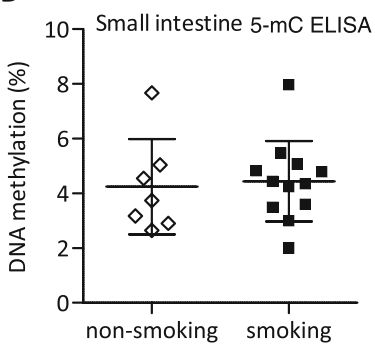

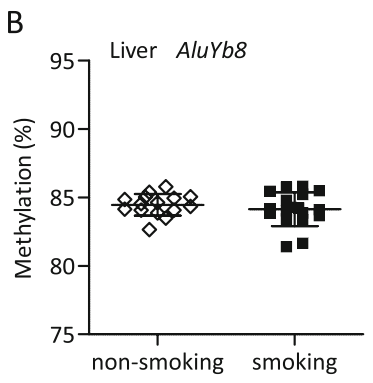

$\mathrm{E}$

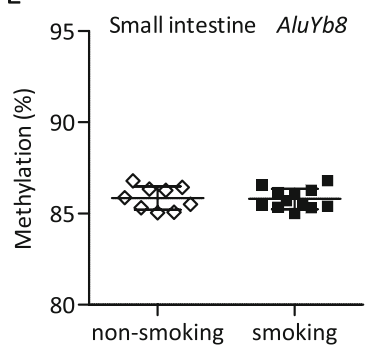

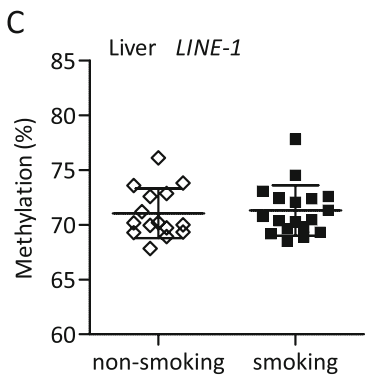

$\mathrm{F}$

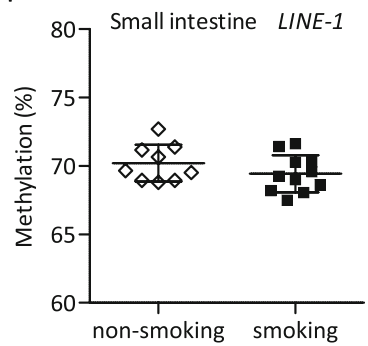

Fig. 2 Global DNA methylation in fetal livers and small intestines. a DNA methylation in the fetal liver was determined by ELISA using 5-mCspecific antibody (non-smoking group $N=10$ and smoking group $N=11$ ). Data represent the means of three experiments. $\mathbf{b}-\mathbf{c}$ Percentage of methylation of AluYb8 and LINE-1 in the fetal liver was determined by pyrosequencing bisulfite-converted DNA (non-smoking group $N=15$ and smoking group $N=18$ ). $\mathbf{d}$ DNA methylation in the fetal small intestine was determined by ELISA using 5 -mC-specific antibody (non-smoking group $N=7$ and smoking group $N=12$ ). Data represent the means of 2 experiments. e-f Percentage of methylation of AluYb8 (non-smoking group $N=9$ and smoking group $N=12$ ) and LINE-1 (non-smoking group $N=9$ and smoking group $N=11$ ) was determined by pyrosequencing bisulfite-converted DNA. Percentages of methylation were calculated, analyzed and displayed as described in the legend for Fig. 1 
methylation levels were not affected by the age of the fetal liver samples (Table 1, ANCOVA).

To investigate another fetal tissue, we also performed DNA methylation analyses of the fetal small intestine. 5$\mathrm{mC}$ ELISA analysis showed no significant changes in the small intestine global DNA methylation levels from PEMCS (Fig. 2d). We also did not detect gender or age effects for the global DNA methylation levels in the fetal small intestines (Table 1 and Additional file 4: Figure S3A). In accordance, bisulfite pyrosequencing of AluYb8 and LINE-1 showed no significant effects for the DNA methylation levels in the small intestines (Fig. 2e and f, Additional file 4: Figure S3B and S3D). Gender and age had no significant effect on the AluYb8 and LINE-1 DNA methylation levels in the small intestines (Table 1 and Additional file 4: Figure S3C and S3E). From the presented results based on the analyses of surrogate markers for global DNA methylation status, we conclude that, at least in the hereby examined sample cohort, the first trimester PEMCS does not affect the global DNA methylation level in fetal small intestines and livers.

\section{Discussion}

In the presented study, we have examined whether PEMCS results in global DNA methylation changes in the first trimester placentas and fetal livers and small intestines. In term placentas and umbilical cord blood, as well as in the peripheral blood and buccal epithelium tissue of children, PEMCS-induced differences in global DNA methylation have previously been described [36, 41, 42]. However, for tissue samples representing early fetal development, to our knowledge, results addressing maternal smoking and global DNA methylation alterations, do not exist, and this lack of information reflects the fact that relevant experimental samples are rarely obtainable. In our analyses examining the first trimester placentas and fetal livers and small intestines, we did not identify significant PEMCS-induced alterations in global DNA methylation levels. This indicates either higher sensitivity for a DNA methylation response later in fetal development or the requirement of a long-term doseeffect response before smoking-mediated DNA methylation alterations will be manifested at birth and in later life [34, 49]. Notably, an immediate manifestation of gene-specific DNA methylation alterations from maternal smoking in the first trimester was shown in the study by Chhabra et al. [39]. Methylation array analyses identified gene-specific DNA methylation alterations associated with nicotine exposure, but only a few of these changes were similar to the DNA methylation changes described to be present at birth, in childhood, or in adulthood due to PEMCS [39]. This observation, as well as our results for global DNA methylation levels, is in contrast to the general assumption that early pregnancy represents the most sensitive period for the lifelong manifestation of environmental-induced epigenetic changes. Chhabra et al.'s [39] observation of gene-specific DNA methylation alterations in the first trimester tissue due to maternal smoking, while global DNA methylation levels were not affected, could reflect the remodeling of specific signaling pathways by maternal cigarette smoking at this particular developmental time. We note the existence of developmental consequences of the first trimester smoking exposure since the number of germ cells is significantly reduced in the first trimester fetuses exposed to maternal cigarette smoke [43].

The relative low number of available fetal samples represents a limitation for the current study since it could prevent the detection of subtle methylation differences; however, we were unable to increase the number of samples given the limited availability of the human samples for our analyses. A possible confounder in studies of the effect of PEMCS is the reliability of self-reported smoking status, as well as exposure to household smoking, which has been found to cause the same DNA methylation effects in newborns as PEMCS [27, 41]. Most women are aware of the fact that smoking during pregnancy can be harmful to their child and may therefore understate their smoking in the mother-child analyses [50-52]. However, as the women included in the present study sought the active termination of their pregnancies, their willingness to report smoking could be more reliable. In support of this, measurements of cotinine levels in similar collected samples from women undergoing the legal termination of their pregnancies have verified the reliability of the women's self-reported smoking habits [43]. In addition, we note that all the non-smoking women in the study reported an absence of exposure to second-hand smoking, whereas nearly all smoking women reported additional exposure to second-hand smoking. Finally, transgenerational smoking effects as well as the preconception smoking of the father and the mother have potential consequences for the fetal epigenome and can confound the methylation levels measured in the non-exposed samples [20].

\section{Conclusion}

Smoking cessation prior to the second trimester of pregnancy seems to have many of the same health benefits as smoking cessation before pregnancy or never having smoked [2, 13, 53, 54]. To the best of our knowledge, the presented data are the first to show that global fetal DNA methylation is not significantly changed in organs from the first trimester PEMCS. Obviously, site-specific CpG methylation alterations from PEMCS with potential devastating health consequences can already be manifested from the first trimester smoking exposure, but not elucidated in the present analyses of global alterations. This was exemplified by Chhabra et al., who 
showed an association between gene-specific DNA methylation changes and nicotine exposure in the first trimester lung and placenta [39]. The current clinical guidelines highlight the importance of the cessation of cigarette smoking before or early in the pregnancy. It is important to stress that epidemiological data and genespecific DNA methylation data still invariably support reinforced attempts of the immediate smoking cessation for women still smoking at the time of pregnancy recognition to improve prenatal care.

\section{Additional files}

Additional file 1: Table S1. Description of fetal and placenta samples The number of samples $(N)$ and fetal age in days are illustrated. Samples were assigned to the smoking-exposed group if mothers smoked one or more cigarettes per day (approximately $90 \%$ of the smoking mothers were smoking 6-20 cigarettes per day). Exposure to passive smoking was not reported among the non-smoking mothers, whereas among the smoking mothers most also reported exposure to passive smoking. Unpaired $t$ test was used to determine whether there is a statistically significant age difference between the non-smoking and smoking groups and between females and males used for a particular assay (LINE-1, AluYb8, and 5-mC ELISA). *Indicates statistical significance, $p<0.05$. (DOCX $17 \mathrm{~kb}$ )

Additional file 2: Figure S1. DNA methylation in placentas. a DNA methylation in female and male fetal placentas determined by the Elisa method using 5-mC-specific antibody (non-smoking female group $N=6$, non-smoking male group $N=9$, smoking female group $N=7$, and smoking male group $N=5$ ). Data represent the mean of two experiments. $\mathbf{b}$ Percentage of AluYb8 DNA methylation at five consecutive CpG sites (non-smoking group $N=19$ and smoking group $N=13$ ). c AluYb8 DNA methylation in males and females (non-smoking female group $N=7$, non-smoking male group $N=12$, smoking female group $N=8$, and smoking male group $N=5$ ). $\mathbf{d}$ Percentage of LINE-1 DNA methylation at three consecutive $\mathrm{CpG}$ sites (non-smoking group $N=22$ and smoking group $N=17$ ). e LINE-1 DNA methylation in males and females (nonsmoking female group $N=9$, non-smoking male group $N=13$, smoking female group $N=9$, and smoking male group $N=8$ ). Percentages of methylation were calculated and displayed as described in the legend for Fig. 1. Unpaired $t$ test was used to compare non-smoking and smoking exposed group, while two-way ANOVA was used to compare combined effects of smoke exposure and gender. *Indicates statistical significance, $p<0.05$. (PDF $559 \mathrm{~kb}$ )

Additional file 3: Figure S2. DNA methylation in fetal livers. a DNA methylation in female and male fetal livers determined by the Elisa method using 5-mC-specific antibody (non-smoking female group $N=4$, non-smoking male group $N=6$, smoking female group $N=6$, and smoking male group $N=5$ ). Data represent the mean of three experiments. b Percentage of AluYb8 DNA methylation at 5 consecutive $C p G$ sites (non-smoking group $N=15$ and smoking group $N=18$ ). c AluYb8 DNA methylation in males and females (non-smoking female group $N=6$, non-smoking male group $N=9$, smoking female group $N=8$, and smoking male group $N=10$ ). $\mathbf{d}$ Percentage of LINE-1 DNA methylation at three consecutive $\mathrm{CpG}$ sites (non-smoking group $N=15$ and smoking group $N=18$ ). e LINE-1 DNA methylation in males and females (non-smoking female group $N=6$, non-smoking male group $N=9$, smoking female group $N=8$, and smoking male group $N=10$ ). Percentages of methylation were calculated and displayed as described in the legend for Fig. 1. Unpaired $t$ test was used to compare non-smoking and smoking exposed group, while two-way ANOVA was used to compare combined effects of smoke exposure and gender. *Indicates statistical significance, $p<0.05$. (PDF $539 \mathrm{~kb}$ )

Additional file 4: Figure S3. DNA methylation in fetal small intestines. a DNA methylation in female and male fetal small intestines determined by the Elisa method using 5-mC-specific antibody (non-smoking female group $N=5$, non-smoking male group $N=2$, smoking female group $N=8$, and smoking male group $N=4$ ). Data represent the mean of two experiments. b Percentage of AluYb8 methylation at 5 consecutive $\mathrm{CpG}$ sites (non-smoking group $N=9$ and smoking group $N=12$ ). c AluYb8 DNA methylation in males and females (non-smoking female group $N=7$, non-smoking male group $N=2$, smoking female group $N=8$, and smoking male group $N=4)$. $\mathbf{d}$ Percentage of LINE-1 DNA methylation at three consecutive CpG sites (non-smoking group $N=9$ and smoking group $N=11$ ). e LINE-1 DNA methylation in males and females (non-smoking female group $N=7$, nonsmoking male group $N=2$, smoking female group $N=7$, and smoking male group $N=4$ ). Percentages of methylation were calculated and displayed as described in the legend for Fig. 1. Unpaired $t$ test was used to compare nonsmoking and smoking exposed group, while two-way ANOVA was used to compare combined effects of smoke exposure and gender. (PDF $529 \mathrm{~kb}$ )

\section{Abbreviations}

5-mC: 5-Methylcytosine; LINE-1: Long interspersed nuclear element-1; PEMCS: Prenatal exposure to maternal cigarette smoking

\section{Acknowledgements}

We thank Sofie Overgaard Pedersen and Mette Høj Skovbo for the help with the DNA purification from the tissue samples and implementing the pyrosequencing assays in the early stage of the project.

\section{Funding}

This work was supported by the Lundbeck Foundation, Dines Hansens Legat, Læge Sofus Carl Emil Friis og Hustru Olga Doris Friis Legat, ReproUnion, and the Toyota Foundation. Svetlana Fa was supported by the Erasmus Mundus JoinEUsee Penta fellowship from the EU.

\section{Availability of data and materials}

Not applicable.

\section{Authors' contributions}

SF, TVL, KB, and TFD performed the molecular epigenetic studies, participated in the data analyses, and drafted the manuscript. SF, RHO, AL, and ALN performed the statistical analyses and prepared the tables and figures. $K B, R H O, E H H, E E, L S M$, and $A L$ collected the samples and the questionnaires. SF, TVL, AL, and ALN designed the molecular analyses and coordinated the study. EE, LSM, AL, and ALN conceived the study. All authors helped draft the manuscript, participated in the manuscript editing, and read/approved the manuscript.

\section{Competing interests}

The authors declare that they have no competing interests.

\section{Consent for publication}

Not applicable.

\section{Ethics approval and consent to participate}

The fetal tissues were obtained with informed consent from women seeking legal (<gestation week 12) abortion in a regional hospital within the Region Midtjylland, Denmark. The study was approved by The Danish National Committee on Health Research Ethics (approval no. KF (01)258206) and all experiments were performed in accordance with the Helsinki Declaration.

\section{Author details}

${ }^{1}$ Department of Biomedicine, Aarhus University, Bartholin building, DK-8000 Aarhus C, Denmark. 'Faculty of Sciences, University of Novi Sad, Novi Sad, Serbia. ${ }^{3}$ Laboratory of Reproductive Biology, The Juliane Marie Centre for Women, Children and Reproduction, University Hospital of Copenhagen, University of Copenhagen, Copenhagen, Denmark. ${ }^{4}$ Department of Obstetrics and Gynecology, University Hospital of Aarhus, Skejby Sygehus, Aarhus, Denmark.

Received: 15 September 2016 Accepted: 18 November 2016 Published online: 25 November 2016 


\section{References}

1. Suter MA, Anders AM, Aagaard KM. Maternal smoking as a model for environmental epigenetic changes affecting birthweight and fetal programming. Mol Hum Reprod. 2013;19(1):1-6.

2. Harrod CS, Reynolds RM, Chasan-Taber L, Fingerlin TE, Glueck DH, Brinton JT, Dabelea D. Quantity and timing of maternal prenatal smoking on neonatal body composition: the Healthy Start study. J Pediatr. 2014;165(4):707-12.

3. Knopik VS, Maccani MA, Francazio S, McGeary JE. The epigenetics of maternal cigarette smoking during pregnancy and effects on child development. Dev Psychopathol. 2012;24(4):1377-90.

4. Ng SP, Zelikoff JT. Smoking during pregnancy: subsequent effects on offspring immune competence and disease vulnerability in later life. Reprod Toxicol. 2007;23(3):428-37.

5. Doherty SP, Grabowski J, Hoffman C, Ng SP, Zelikoff JT. Early life insult from cigarette smoke may be predictive of chronic diseases later in life. Biomarkers. 2009;14 Suppl 1:97-101.

6. Carlsen KH, Lodrup Carlsen KC. Parental smoking and childhood asthma: clinical implications. Treat Respir Med. 2005;4(5):337-46.

7. Burke H, Leonardi-Bee J, Hashim A, Pine-Abata H, Chen Y, Cook DG, Britton JR, McKeever TM. Prenatal and passive smoke exposure and incidence of asthma and wheeze: systematic review and meta-analysis. Pediatrics. 2012; 129(4):735-44.

8. Wang L, Pinkerton KE. Detrimental effects of tobacco smoke exposure during development on postnatal lung function and asthma. Birth Defects Res C Embryo Today. 2008;84(1):54-60.

9. Duijts L. Fetal and infant origins of asthma. Eur J Epidemiol. 2012;27(1):5-14.

10. Eskenazi B, Castorina R. Association of prenatal maternal or postnatal child environmental tobacco smoke exposure and neurodevelopmental and behavioral problems in children. Environ Health Perspect. 1999;107(12):991-1000.

11. Agrawal A, Scherrer JF, Grant JD, Sartor CE, Pergadia ML, Duncan AE, Madden PA, Haber JR, Jacob T, Bucholz KK, et al. The effects of maternal smoking during pregnancy on offspring outcomes. Prev Med. 2010;50(1-2):13-8.

12. Morris CV, DiNieri JA, Szutorisz H, Hurd YL. Molecular mechanisms of maternal cannabis and cigarette use on human neurodevelopment. Eur J Neurosci. 2011:34(10):1574-83.

13. Ino $T$, Shibuya $T$, Saito $K$, Inaba $Y$. Relationship between body mass index of offspring and maternal smoking during pregnancy. Int J Obes (Lond). 2012; 36(4):554-8

14. Oken E, Levitan EB, Gillman MW. Maternal smoking during pregnancy and child overweight: systematic review and meta-analysis. Int J Obes (Lond). 2008:32(2):201-10.

15. Banderali G, Martelli A, Landi M, Moretti F, Betti F, Radaelli G, Lassandro C, Verduci E. Short and long term health effects of parental tobacco smoking during pregnancy and lactation: a descriptive review. J Transl Med. 2015;13:327.

16. Oken E, Huh SY, Taveras EM, Rich-Edwards JW, Gillman MW. Associations of maternal prenatal smoking with child adiposity and blood pressure. Obes Res. 2005;13(11):2021-8.

17. Hogberg L, Cnattingius S, Lundholm C, D'Onofrio BM, Langstrom N, Iliadou AN. Effects of maternal smoking during pregnancy on offspring blood pressure in late adolescence. J Hypertens. 2012;30(4):693-9.

18. Cohen G, Jeffery H, Lagercrantz H, Katz-Salamon M. Long-term reprogramming of cardiovascular function in infants of active smokers. Hypertension. 2010;55(3):722-8

19. Geerts CC, Grobbee DE, van der Ent CK, de Jong BM, van der Zalm MM, van Putte-Katier N, Kimpen JL, Uiterwaal CS. Tobacco smoke exposure of pregnant mothers and blood pressure in their newborns: results from the wheezing illnesses study Leidsche Rijn birth cohort. Hypertension. 2007; 50(3):572-8.

20. Nielsen $\mathrm{CH}$, Larsen $\mathrm{A}$, Nielsen AL. DNA methylation alterations in response to prenatal exposure of maternal cigarette smoking: a persistent epigenetic impact on health from maternal lifestyle? Arch Toxicol. 2016;90(2):231-45.

21. Swanson JM, Entringer S, Buss C, Wadhwa PD. Developmental origins of health and disease: environmental exposures. Semin Reprod Med. 2009; 27(5):391-402

22. Szyf M, Bick J. DNA methylation: a mechanism for embedding early life experiences in the genome. Child Dev. 2013;84(1):49-57.

23. Wadhwa PD, Buss C, Entringer S, Swanson JM. Developmental origins of health and disease: brief history of the approach and current focus on epigenetic mechanisms. Semin Reprod Med. 2009:27(5):358-68.
24. Slieker RC, Roost MS, van Iperen L, Suchiman HE, Tobi EW, Carlotti F, de Koning EJ, Slagboom PE, Heijmans BT, Chuva de Sousa Lopes SM. DNA methylation landscapes of human fetal development. PLoS Genet. 2015; 11(10):e1005583.

25. Tomizawa S, Nowacka-Woszuk J, Kelsey G. DNA methylation establishment during oocyte growth: mechanisms and significance. Int J Dev Biol. 2012; 56(10-12):867-75

26. Joubert BR, Haberg SE, Bell DA, Nilsen RM, Vollset SE, Midttun O, Ueland PM, Wu MC, Nystad W, Peddada SD, et al. Maternal smoking and DNA methylation in newborns: in utero effect or epigenetic inheritance? Cancer Epidemiol Biomarkers Prev. 2014;23(6):1007-17.

27. Suter MA, Aagaard K. What changes in DNA methylation take place in individuals exposed to maternal smoking in utero? Epigenomics. 2012;4(2):115-8.

28. Maccani JZ, Maccani MA. Altered placental DNA methylation patterns associated with maternal smoking: current perspectives. Adv Genomics Genet. 2015;2015(5):205-14.

29. Pirini F, Guida E, Lawson F, Mancinelli A, Guerrero-Preston R. Nuclear and mitochondrial DNA alterations in newborns with prenatal exposure to cigarette smoke. Int J Environ Res Public Health. 2015:12(2):1135-55.

30. Lee KW, Richmond R, Hu P, French L, Shin J, Bourdon C, Reischl E, Waldenberger M, Zeilinger S, Gaunt T, et al. Prenatal exposure to maternal cigarette smoking and DNA methylation: epigenome-wide association in a discovery sample of adolescents and replication in an independent cohort at birth through 17 years of age. Environ Health Perspect. 2015;123(2):193-9.

31. Joubert BR, Haberg SE, Nilsen RM, Wang X, Vollset SE, Murphy SK, Huang Z, Hoyo C, Midttun O, Cupul-Uicab LA, et al. 450K epigenome-wide scan identifies differential DNA methylation in newborns related to maternal smoking during pregnancy. Environ Health Perspect. 2012;120(10):1425-31.

32. Joubert BR, Felix JF, Yousefi P, Bakulski KM, Just AC, Breton C, Reese SE, Markunas CA, Richmond RC, Xu CJ, et al. DNA methylation in newborns and maternal smoking in pregnancy: genome-wide consortium meta-analysis. Am J Hum Genet. 2016;98(4):680-96.

33. Morales E, Vilahur N, Salas LA, Motta V, Fernandez MF, Murcia M, Llop S, Tardon A, Fernandez-Tardon G, Santa-Marina L, et al. Genome-wide DNA methylation study in human placenta identifies novel loci associated with maternal smoking during pregnancy. Int J Epidemiol. 2016.

34. Richmond RC, Simpkin AJ, Woodward G, Gaunt TR, Lyttleton O, McArdle WL, Ring SM, Smith AD, Timpson NJ, Tilling K, et al. Prenatal exposure to maternal smoking and offspring DNA methylation across the lifecourse: findings from the Avon Longitudinal Study of Parents and Children (ALSPAC). Hum Mol Genet. 2015;24(8):2201-17.

35. Guerrero-Preston R, Herbstman J, Goldman LR. Epigenomic biomonitors: global DNA hypomethylation as a biodosimeter of life-long environmental exposures. Epigenomics. 2011:3(1):1-5.

36. Wilhelm-Benartzi CS, Houseman EA, Maccani MA, Poage GM, Koestler DC, Langevin SM, Gagne LA, Banister CE, Padbury JF, Marsit CJ. In utero exposures, infant growth, and DNA methylation of repetitive elements and developmentally related genes in human placenta. Environ Health Perspect. 2012:120(2):296-302

37. Choi SH, Worswick S, Byun HM, Shear T, Soussa JC, Wolff EM, Douer D, Garcia-Manero G, Liang G, Yang AS. Changes in DNA methylation of tandem DNA repeats are different from interspersed repeats in cancer. Int J Cancer. 2009:125(3):723-9.

38. Michels KB, Harris HR, Barault L. Birthweight, maternal weight trajectories and global DNA methylation of LINE-1 repetitive elements. PLoS One. 2011;6(9), e25254.

39. Chhabra D, Sharma S, Kho AT, Gaedigk R, Vyhlidal CA, Leeder JS, Morrow J, Carey VJ, Weiss ST, Tantisira KG, et al. Fetal lung and placental methylation is associated with in utero nicotine exposure. Epigenetics. 2014;9(11):1473-84.

40. Suter M, Ma J, Harris A, Patterson L, Brown KA, Shope C, Showalter L, Abramovici A, Aagaard-Tillery KM. Maternal tobacco use modestly alters correlated epigenome-wide placental DNA methylation and gene expression. Epigenetics. 2011;6(11):1284-94.

41. Guerrero-Preston R, Goldman LR, Brebi-Mieville P, Ili-Gangas C, Lebron C, Witter FR, Apelberg BJ, Hernandez-Roystacher M, Jaffe A, Halden RU, et al. Global DNA hypomethylation is associated with in utero exposure to cotinine and perfluorinated alkyl compounds. Epigenetics. 2010;5(6):539-46.

42. Breton CV, Byun HM, Wenten M, Pan F, Yang A, Gilliland FD. Prenatal tobacco smoke exposure affects global and gene-specific DNA methylation. Am J Respir Crit Care Med. 2009:180(5):462-7. 
43. Mamsen LS, Lutterodt MC, Andersen EW, Skouby SO, Sorensen KP, Andersen $C Y$, Byskov AG. Cigarette smoking during early pregnancy reduces the number of embryonic germ and somatic cells. Hum Reprod. 2010;25(11):2755-61.

44. Li S, Feng T, Fu L, Li Z, Lou C, Zhang X, Ma C, Cong B. Pyrosequencing of a short fragment of the amelogenin gene for gender identification. Mol Biol Rep. 2012;39(6):6949-57.

45. Liu F, Killian JK, Yang M, Walker RL, Hong JA, Zhang M, Davis S, Zhang Y, Hussain $\mathrm{M}, \mathrm{Xi}$ S, et al. Epigenomic alterations and gene expression profiles in respiratory epithelia exposed to cigarette smoke condensate. Oncogene. 2010;29(25):3650-64.

46. Murphy SK, Adigun A, Huang Z, Overcash F, Wang F, Jirtle RL, Schildkraut JM, Murtha AP, Iversen ES, Hoyo C. Gender-specific methylation differences in relation to prenatal exposure to cigarette smoke. Gene. 2012;494(1):36-43.

47. Breton CV, Salam MT, Gilliland FD. Heritability and role for the environment in DNA methylation in AXL receptor tyrosine kinase. Epigenetics. 2011;6(7):895-8.

48. Drake AJ, O'Shaughnessy PJ, Bhattacharya S, Monteiro A, Kerrigan D, Goetz S, Raab A, Rhind SM, Sinclair KD, Meharg AA, et al. In utero exposure to cigarette chemicals induces sex-specific disruption of one-carbon metabolism and DNA methylation in the human fetal liver. BMC Med. 2015;13:18.

49. Novakovic B, Ryan J, Pereira N, Boughton B, Craig JM, Saffery R. Postnatal stability, tissue, and time specific effects of AHRR methylation change in response to maternal smoking in pregnancy. Epigenetics. 2014;9(3):377-86.

50. Dietz PM, Homa D, England L, Burley K, Tong VT, Dube SR, Bernert JT. Estimates of nondisclosure of cigarette smoking among pregnant and nonpregnant women of reproductive age in the United States. Am J Epidemiol. 2011;173(3):355-9.

51. Shipton D, Tappin DM, Vadiveloo T, Crossley JA, Aitken DA, Chalmers J. Reliability of self reported smoking status by pregnant women for estimating smoking prevalence: a retrospective, cross sectional study. BMJ. 2009;339:b4347.

52. George L, Granath F, Johansson AL, Cnattingius S. Self-reported nicotine exposure and plasma levels of cotinine in early and late pregnancy. Acta Obstet Gynecol Scand. 2006;85(11):1331-7.

53. Vardavas Cl, Chatzi L, Patelarou E, Plana E, Sarri K, Kafatos A, Koutis AD, Kogevinas M. Smoking and smoking cessation during early pregnancy and its effect on adverse pregnancy outcomes and fetal growth. Eur J Pediatr. 2010;169(6):741-8.

54. England L, Kendrick JS, Wilson HG, Merritt RK, Gargiullo PM, Zahniser SC Effects of smoking reduction during pregnancy on the birth weight of term infants. Am J Epidemiol. 2001;154(8):694-701.

\section{Submit your next manuscript to BioMed Central and we will help you at every step:}

- We accept pre-submission inquiries

- Our selector tool helps you to find the most relevant journal

- We provide round the clock customer support

- Convenient online submission

- Thorough peer review

- Inclusion in PubMed and all major indexing services

- Maximum visibility for your research

Submit your manuscript at www.biomedcentral.com/submit

) Biomed Central 